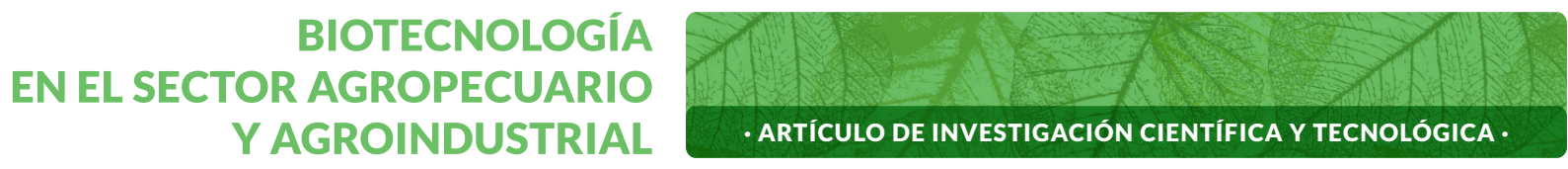

Vol. 20 No 1 · Enero-Junio 2022 · ISSN-1692-3561 · ISSN-e 1909-9959· DOI: https://doi.org/10.18684/rbsaa.v20.n1.2022.1914

\title{
Identificación molecular de hongos filamentosos y su potencial biotecnológico*
}

\section{Molecular identification of filamental fungi and its biotechnological potential}

\author{
SUÁREZ-CONTRERAS, LILIANA-YANET'1; PEÑARANDA-FIGUEREDO, FREDDY-ARMANDO²
}

\begin{abstract}
RESUMEN
Los hongos filamentosos son organismos frecuentemente utilizados en el desarrollo de alternativas para resolver desafíos en diferentes sectores productivos. Igualmente, representan una oportunidad competitiva de valor económico en sectores como el agrícola, alimenticio y farmacéutico como también en el industrial y de biocombustibles. Dando continuidad a investigaciones previas de la Universidad Francisco de Paula Santander (UFPS), se analizaron 25 muestras del banco de cepas para caracterización molecular e identificación taxonómica. Se obtuvo ADN de cada cepa con la técnica fenol-clo-
\end{abstract}

Historial del artículo

Recibido para evaluación: 23 Abril 2021.

Aprobado para publicación: 10 Noviembre 2021.

* $\quad$ Proyecto de investigación de origen: "Identificación molecular de los aislamientos de hongos conservados en el banco de cepas de la Facultad de Ciencias Agrarias y del Ambiente de la Universidad Francisco de Paula Santander". Financiación: COLCIENCIAS-UFPS, convocatoria 753-2016. Finalización: mayo 30 de 2018.

1 Universidad Francisco de Paula Santander, Facultad de Ciencias Agrarias y del Ambiente, Grupo de investigación Ambiente y Vida. Magíster en Biología. Cúcuta, Colombia. https://orcid.org/0000-0001-8020-502X

2 Universidad Francisco de Paula Santander, Facultad de Ciencias Agrarias y del Ambiente, Grupo de investigación Ambiente y Vida. Ingeniero Biotecnológico. Cúcuta, Colombia. https://orcid.org/0000-0002-0008-0667

Correspondencia: lilianayanethsc@ufps.edu.co

Cómo citar este artículo: SUÁREZ-CONTRERAS, LILIANA-YANET; PEÑARANDA-FIGUEREDO, FREDDY-ARMANDO. Identificación molecular de hongos filamentosos y su potencial biotecnológico. Revista Biotecnología en el Sector Agropecuario y Agroindustrial, v. 20, n. 1, 2022, p. 194-206. Doi: https://doi.org/10.18684/rbsaa.v20.n1.2022.1914 
roformo. Mediante las técnicas de PCR y de iniciadores de espaciadores internos de transcrito (ETS) primer 4 y 5 , se obtuvieron los amplicones que fueron secuenciados. Se identificaron cepas de los ordenes Hypocreales, Eurotiales, Pleosporales y Saccharomycetales con ejemplares de géneros como el Aspergillus, Curvularia; Purpureocillium, Penicillium y Trichoderma entre otros. Los hallazgos se exhiben en el cladograma evidenciando la proximidad filogenética como también relacionando los potenciales biotecnológicos para el desarrollo de bio insumos, productos farmacéuticos y biocatalizadores. Gracias al estudio y revisión en bases de datos se logró avanzar en la descripción de las capacidades biológicas de estos hongos hacia el desarrollo de productos o servicios con base biotecnológica y enfoque de investigación en fitopatología.

\section{ABSTRACT}

Filamentous fungi are organisms frequently used to develop alternatives to solve challenges in different productive representing a competitive opportunity with economic value in the agriculture, food and pharmaceutical, as well as industrial and biofuels sector Continuing previous research at Universidad Francisco de Paula Santander (UFPS), 25 samples from the strain bank were used for molecular characterization and taxonomic identification. DNA from each strain was obtained under the phenol-chloroform procedure, then, using PCR technique and internal transcript spacer (ITS) primer 4 and 5, amplicons were acquired to be sequenced. Results showed taxonomy categorizations in 4 orders Hypocreales, Eurotiales, Pleosporales y Saccharomycetales with specimens from genus like Aspergillus, Curvularia; Purpureocillium, Penicillium y Trichoderma among others. Findings were laid into a cladogram to display their phylogenetic proximity, and connecting their biotechnological potential towards developing bio-materials, pharmaceutical products and bio-catalysts. Thanks to present study and data base review, it was possible to advance into describing the biological capabilities of these fungi towards developing biotechnological products and services with an approach in phytopathology research.

\section{PALABRAS CLAVES:}

ADN; Bioprospección; Cladograma; Control biológico; Homología; ETS; Marcador molecular; Mohos; Patógeno; PCR.

\section{KEYWORDS:}

DNA; Bioprospecting; Cladogram; Biologic control; Homology; ITS; Molecular marker; Molds; Pathogen; PCR

\section{INTRODUCCIÓN}

Los hongos filamentosos o mohos se caracterizan por tener un soma vegetativo (talo) similar a las plantas, filamentos microscópicos continuos más o menos alargados y ramificados con paredes celulares definidas, la mayoría, constituidas por quitina, dispuestas en microfibrillas como la celulosa, además de otros polisacáridos como mananos, galactanos y quitosán reemplazan a la quitina en algunos grupos, con una la pared celular formada por carbohidratos (80-90\%), y son las proteínas, los lípidos, polifosfatos e iones inorgánicos el material cementante (Estrada et al., 2019).

Muchos hongos son beneficiosos, al degradar la materia orgánica compleja convirtiéndola a formas químicas simples que pasan a formar parte del suelo, absorbidas por otras generaciones de plantas, encargándose de la fertilidad de la tierra. Pese a los perjuicios que pueden causar estos organismos a 
otros seres vivos, incluyendo a humanos, son un grupo de organismos muy importantes y exitosos desde el punto de vista evolutivo trascendental para la vida en el planeta (Tellez et al., 2017; Mancilla et al., 2021).

Otros hongos filamentosos tienen potencial de aplicación biotecnológica en diferentes sectores productivos debido a su capacidad para producir antioxidantes (Smith et al., 2015), ácidos grasos polinsaturados, enzimas industriales, antibióticos, productos fermentados (Wakai et al., 2017), pigmentos que se utilizan en alimentos (Dufossé et al., 2014), antifúngicos, agentes hipolipidémico e inmunosupresores (Alberti et al., 2017), y debido a su capacidad metabólica y mecánica diferentes especies de mohos se utilizan para el desarrollo de biofertilizantes, biopesticidas y acondicionadores biológicos (Tellez et al., 2017). También, la necesidad de afianzar el crecimiento de los sistemas productivos agrícolas ha creado alterativas permitiendo avanzar y superar las deficiencias en procesos como la nutrición vegetal a partir del uso de fertilizantes de síntesis. Así, ha surgido la agricultura orgánica como una tecnología eficaz, respetuosa del medio ambiente, económica y factible de desarrollar, incluyendo la producción y uso de fertilizantes de origen microbiológico a partir de residuos agropecuarios para nutrir plantas, controlar plagas y enfermedades de plantas, e influir positivamente en las propiedades fisicoquímicas del suelo (Rojas, 2020).

Definir la identidad de las cepas es una fase insustituible en la investigación de cualquier agente biológico y la selección de las herramientas y mecanismo de identificación constituyen la confiabilidad de la información. El uso de la técnica de la Reacción de la Cadena de la Polimerasa (PCR) como mecanismo de identificación molecular se precisa debido a la fiabilidad y practicidad de la técnica para amplificar segmentos de ADN asistido por cebadores como los Espaciadores internos de transcrito (ITS) que conforman la región ITS que se caracteriza por ser altamente repetitiva dentro del ADNr, poseer regiones conservadas, tener evolución relativamente lenta, pero a su vez, contener regiones menos conservadas, propiedades otorgadas por ITS2, 5.8 S e ITS1 respectivamente (Froeschke at al., 2014); dichas características hacen de la región ITS un segmento significativamente de interés para estudios en taxonomía, incluso el espaciador interno de transcrito ha llegado a ser denominado por un grupo de micólogos como el marcador de barra para identificación de taxones y constituye uno de los mecanismos más confiables para determinar la identidad de mohos a nivel de especie (Bellemain et al., 2010).

Esta investigación determinó un mecanismo de identificación molecular para doce especies de hongos distribuidos en los géneros Trichoderma, Geotrichum, Purpureocillium, Laburnicola, Penicillium, Aspergillus, Beauveria, Bipolaris, Curvularia, y Alternaria. Además, se realizó una correlación filogenética y revisión de antecedentes que enlistan una serie de capacidades de las diferentes especies identificadas para futuras aplicaciones en diferentes áreas de biotecnología. También, importante tanto para el banco de cepas como para el laboratorio de biotecnología molecular de la sede campos Elíseos de la Universidad Francisco de Paula Santander que permitirá avances en procesos de docencia e investigación.

\section{MÉTODO}

Esta investigación se realizó en el Centro Experimental Campos Elíseos, de la Facultad de Ciencias Agrarias y del Ambiente de la Universidad Francisco de Paula Santander, ubicado en el municipio de Los Patios, Norte de Santander (Colombia) a una altura de 410 ms.n.m., y temperatura de $27^{\circ} \mathrm{C}$. Los aislamientos de los hongos conservados fueron caracterizados y procesados en los laboratorios Banco de Cepas y Biotecnología Molecular. 


\section{Extracción de ADN}

Posterior al crecimiento en caldo Sabouraud, el micelio fue fraccionado con ayuda de una cuchilla de bisturí en trozos con diámetro no superior a 4 mm², exceptuando las cepas HA002, HE004, HF002 y HF003 a las que el micelio se centrifugó a 5000 rpm durante 5 minutos, y se procedió utilizando los protocolos propuestos por Suárez (2016) y Blanco et al. (2021).

\section{Obtención de los amplicones}

Para la obtención de los fragmentos amplificados por medio de la PCR se utilizaron los cebadores ITS5 (5'-GGAAGTAAAAGTCGTAACAAGG-3') e ITS4 (5'-TCCTCCGCTTATTGATATGC-3') que amplificaron la región ITS en sentido y anti-sentido respectivamente, haciendo uso del protocolo propuesto por (Suárez, 2016).

\section{Visualización y cuantificación de los amplicones de ADN}

Para la visualización de los fragmentos de ADN amplificados se realizó en gel de agarosa al 1,5 \% w/v y tampón TBE 1X, y las electroforesis se efectuó con un arranque de corrida de 120 voltios durante 5 minutos y posteriormente a 100 voltios durante 2 horas. Las muestras de ácidos nucleicos fueron marcadas con el intercalante gel red y revelado en el transiluminador y fotodocumentador ChemiDoc de Bio-Rad. Para la cuantificación de ADN se utilizó el espectrofotómetro NanoDrop de Thermo scientific (Suárez, 2005).

\section{Secuenciación de amplicones}

Los productos de PCR obtenidos de cada una de las cepas se enviaron a la empresa Isla SAS, (Corea del Sur) para la posterior decodificación de las secuencias amplificadas, utilizando el secuenciador 3730xI DNA Analyzer. Los resultados fueron recibidos en formato fasta y la lectura del secuenciador fue reflejado en un archivo por medio de un cromatograma.

\section{Identidad por homología}

A partir de las secuencias obtenidas, se realizó un análisis utilizando la base de datos del Centro Nacional de Información Biotecnológica (NCBI, 2019) mediante la herramienta Targeted Loci Nucleotide BLAST que permitió determinar la identidad de los organismos mediante la comparación de la secuencia target (mi secuencia de interés) con la base de datos específica para secuencias curadas y remarcadas de la región espaciadora transcrita interna (ITS). Para ello se cargó la secuencia en formato fasta en la interfaz del Blast Targeted Loci Nucleotide, se seleccionó la base de datos para ITS sin ninguna otra modificación de parámetro, y se dio la orden de cargar el análisis. Como resultado se obtuvo la información de las secuencias depositadas en la base de datos con alineaciones significativas, teniendo en cuenta especialmente dos parámetro para la elección de la identidad: el E-value, cuyo valor debe estar próximo a un puntaje de 0,0 y la identidad con un porcentaje del 90 al $100 \%$.

\section{Representación de la relación filogenética}

Para la construcción del árbol filogenético se utilizó el software Mega 7,0; inicialmente, se realizó un alineamiento múltiple entre todas las secuencias incluida el outgrup (E. coli), se empleó el algoritmo de Muscle (Align by Muscle) en Mega 7,0, utilizando el método de agrupamiento UPGMA (Unweighted Pair-Group Method with Arithmetic); una vez culminado el análisis, el archivo que se generó fue guardado en formato Mega y se indicó que no correspondía a secuencias que codifican para proteínas, a partir del archivo de alineamiento múltiple se construyó el dendrograma en la opción phylogeny; para la reconstrucción de la filogenia se empleó el método estadístico de UPGMA, y el modelo Jukes-Cantor del software mencionado, logrando la representación del árbol inferido para la relación filogenética de las secuencias obtenidas en este estudio (Lessick, 2016). 


\section{RESULTADOS}

\section{Amplicones ITS mediante PCR}

Con el protocolo utilizado en la PCR y con la implementación de los cebadores ITS4 e ITS5, se obtuvieron fragmentos de tamaños que variaron entre 0,5 y 0,8 Kilobases (Kb) (Figura 1), permitiendo un testeo de los amplicones obtenidos. A partir de este resultado se define lo que representa el fundamento de selección del proceso estándar para la identificación molecular de hongos filamentosos implementado por el Laboratorio de Biotecnología Molecular de la UFPS. Además, según González-Estrada et al. (2020), para la identificación molecular de Penicillium sp. fue también realizada por PCR, empleando las regiones internas transcritas ITS1-5.8S-ITS2, utilizando el par de iniciadores de secuencia ITS4/ ITS5, y los pares de base obtenidos se compararon con las secuencias reportadas en la base de datos del banco de genes de NCBI mediante el programa BLAST; además, Bejár et al. (2019), amplificaron ADN por PCR en tiempo real (qPCR), para identificar Aspergillus fumigatus.

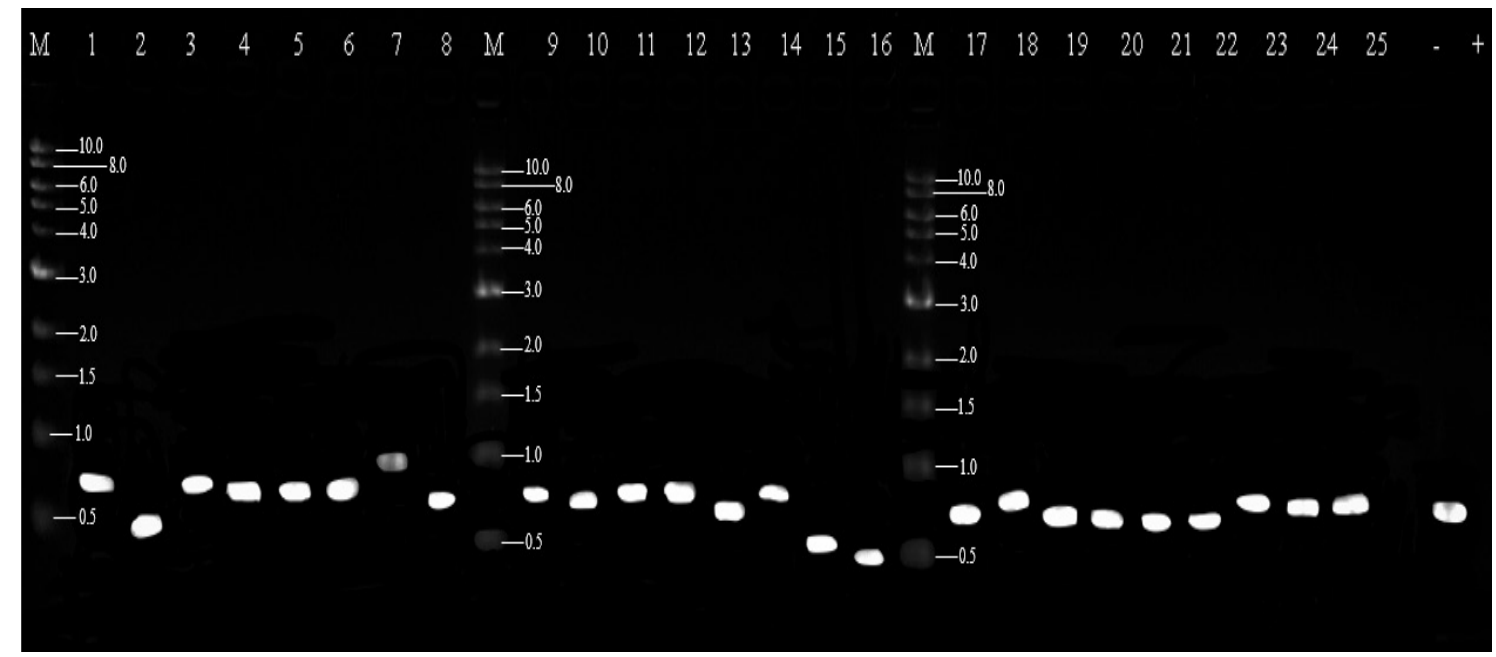

Figura 1. Electroforesis en gel de agarosa al 1,5\% w/v, de los amplicones obtenidos a partir del ADN de hongos, utilizando ITS. M: Marcador molecular. 1, HA001; 2, HA002; 3, HA005; 4, HA006; 5, HA007; 6, HA008; 7, HD002; 8, HD008; 9, HD010; 10, HD011; 11, HE001; 12, HE002; 13, HE003; 14, HE004; 15, HF002; 16, HF003; 17, HFO20; 18, HF021; 19, HI001; 20, HIOO2; 21, HIOO3; 22, HI004; 23, HI005; 24, JIF01; 25, JIFO2; control (-); control (+), Baeauveria bass.

\section{Determinación de identidad de hongos}

Las 25 cepas se clasificaron en diez géneros identificados como Trichoderma, Geotrichum, Purpureocillum, Luburnicula, Penicillum, Aspergillus, Baeuveria, Bipolaris, Curvularia y Alternaria, distribuidos en doce (12) especies, de la siguiente manera: las cepas HA001, HA005 y HA006 corresponden a Trichoderma yunnanense; HA002, HE004, HF002 y HF003 son Geotrichum silvícola; HA007, HA008 y HE003 son Purpureocillium lilacinum; HD011, HI001, HI002, HI003, HI004 y HI005 corresponden a Penicillium citrinum; JIF01 y JIF02 son Alternaria destruens, la cepa HD002 es Laburnicola hawksworthii; HD008 es Penicillium chrysogenum; HD010 corresponde a Aspergillus fumigatus; HE001 a Penicillium rubens; HE002 es Beauveria bassiana; HF020 pertenece a Bipolaris sivanesaniana, y para finalizar HF021 es Curvularia pisi (Cuadro 1). 
Cuadro 1. Identificación, valores de identidad y E-value de cada una de las secuencias analizadas utilizando la base de datos NCBI.

\begin{tabular}{|c|c|c|c|c|c|c|c|}
\hline Cód & Especies & Evalue & $\begin{array}{c}\% \\
\text { Ident }\end{array}$ & Cód & Especies & $\begin{array}{c}E \\
\text { value }\end{array}$ & \% Ident \\
\hline HA001 & Trichoderma yunnanense & 0,0 & $99 \%$ & HE004 & Geotrichum silvicola & $8,00 E-135$ & $94 \%$ \\
\hline HA002 & Geotrichum silvicola & $5,00 \mathrm{E}-147$ & $95 \%$ & HFO02 & Geotrichum silvicola & $4,00 \mathrm{E}-144$ & $95 \%$ \\
\hline HA005 & Trichoderma yunnanense & 0,0 & $99 \%$ & HF003 & Geotrichum silvicola & $3,00 E-143$ & $95 \%$ \\
\hline HA006 & Trichoderma yunnanense & 0,0 & $99 \%$ & HF020 & Bipolaris sivanesaniana & 0,0 & $99 \%$ \\
\hline HA007 & Purpureocillium lilacinum & 0,0 & $99 \%$ & \begin{tabular}{|l|} 
HF021 \\
\end{tabular} & Curvularia pisi & 0,0 & $98 \%$ \\
\hline HA008 & Purpureocillium lilacinum & 0,0 & $99 \%$ & HI001 & Penicillium citrinum & 0,0 & $99 \%$ \\
\hline HD002 & Laburnicola hawksworthii & 8,00E-13 & $92 \%$ & HIOO2 & Penicillium citrinum & 0,0 & $99 \%$ \\
\hline HD008 & Penicillium chrysogenum & 0,0 & $99 \%$ & HIOO3 & Penicillium citrinum & 0,0 & $99 \%$ \\
\hline HD010 & Aspergillus fumigatus & 0,0 & $99 \%$ & HI004 & Penicillium citrinum & 0,0 & $99 \%$ \\
\hline HD011 & Penicillium citrinum & 0,0 & $99 \%$ & HI005 & Penicillium citrinum & 0,0 & $99 \%$ \\
\hline HE001 & Penicillium rubens & 0,0 & $97 \%$ & JIF01 & Alternaria destruens & 0,0 & $93 \%$ \\
\hline HE002 & Beauveria bassiana & 0,0 & $98 \%$ & JIF02 & Alternaria destruens & 0,0 & $99 \%$ \\
\hline HE003 & Purpureocillium lilacinum & $7,00 \mathrm{E}-173$ & $90 \%$ & & & & \\
\hline
\end{tabular}

Los valores de identidad para todas las cepas superaron el $93 \%$ y con E-value no inferior a 8×10-135, según los análisis realizados a las 50 secuencias utilizando la herramienta Targeted Loci Nucleotide BLAST exceptuando la cepa HD002 cuyo resultado fue inconcluso en la secuencia ITS4, mientras que con el ITS5 el valor de identidad alcanzó el 92 \% relacionando la especie Laburnicola hawksworthii.

Se considera la importancia de los datos moleculares para estudios de biodiversidad y el potencial de la investigación micológica en países como Brasil y Colombia. Para el caso de Brasil se determinó la diversidad de hongos basada en las secuencias ITS con un límite del 98 \% (Menolli, 2020), mientras que en este trabajo fue del 97 al $99 \%$.

Aunque los niveles de identidad son significativos y, a pesar de que la región ITS es considerada un código de barras para la identificación taxonómica de hongos (Melloni, 2020), el uso estándar de estos cebadores durante la PCR puede generar sesgos, incurriendo al error en la secuencia, generando una predisposición en la clasificación de especies dentro de las divisiones basidiomicetos y ascomicetos, dependiendo de los iniciadores utilizados (Bellemain et al., 2010). Por lo anteriormente señalado, se sugiere evaluar otros iniciadores para comparar los resultados y establecer un consenso mediante el análisis de las secuencias obtenidas por Bejár et al. (2019), logrando identificar diez cepas de Aspergillus fumigatus sensu stricto Involucradas en la aspergilosis invasiva (AI) en el Perú, contribuyendo a explicar la resistencia al tratamiento y evidenciando la importancia del ambiente en el incremento de los casos.

\section{Análisis filogenético}

El filo Ascomycota se caracteriza por su estructura sexual en forma de saco o asca que contienen las ascosporas y por la conjugación de hifas asimilativas por medio de la anastomosis, también se constituye como un grupo de hongos numeroso con aproximadamente 114.000 especies identificadas y distribuidas en diversos ecosistemas (Wijayawardene et al., 2021). Basados en la información extraída del repositorio de nomenclatura Index Fungorum (http://www.indexfungorum.org/names/Names.asp) la totalidad de las cepas identificadas en el presente estudio corresponden al filo Ascomycota, representando los subfilos Pezizomycotina y Saccharomycotina, enclaustrados en las clases Sordariomycetes, Eurotiomycetes, Dothideomycetes y Saccharomycetes, y asociados a los órdenes Hypocreales, Eurotiales, Pleosporales y Saccharomycetales. Las especies Trichoderma yunnanense, PurpureociIlum lilacinum y Beauveria bassiana son hongos que se caracterizan por su efecto antagonista y son ampliamente utilizados para el control biológico (Hernández et al., 2019; Rui et al., 2020; Romero et al., 2020) y pertenecen al orden de los Hypocreales; Aspergillus fumigatus, Penicillium chrysogenum, Penicillium Rubens y Penicillium citrinum son especies productoras de metabolitos de uso farmacéutico y reconocidos reductores de azúcares de interés 
para la industria de los biocombustibles y hacen parte del grupo de los Eurotiales (Cabral et al., 2018; Ouephanit et al., 2019; Lina et al., 2019; Tejas et al., 2019; Lodha et al., 2020; Huber et al., 2020; Xianchun et al., 2020; Mondal et al., 2020); Bipolaris sivanesaniana, Laburnicola hawksworthii, Alternaria destruens y Curvularia pisi son especies vinculadas con efectos patógenos en diferencies especies vegetales (Manamgoda et al., 2014; Zhai et al., 2018) y son del orden de los Pleosporales; y en el orden de los Saccharomycetales se encuentra a Geotrichum silvícola.

En cuanto al cladograma (Figura 2) se puede inferir la relación entre los diferentes órdenes representado en las bifurcaciones que constituyeron clados entre Hypocreales - Eurotiales, Hypocreales - Pleosporales, Eurotiales - Pleosporales, Hypocreales - Eurotiales - Pleosporales - Saccharomycetales, respaldando los resultados obtenidos por Wang et al. (2009), que señalan la proximidad taxonómica entre los subfilos Pezizomycotina y Saccharomycotina basados en estudios de filogenia y que sugiere una filiación temprana entre los subfilos señalados.

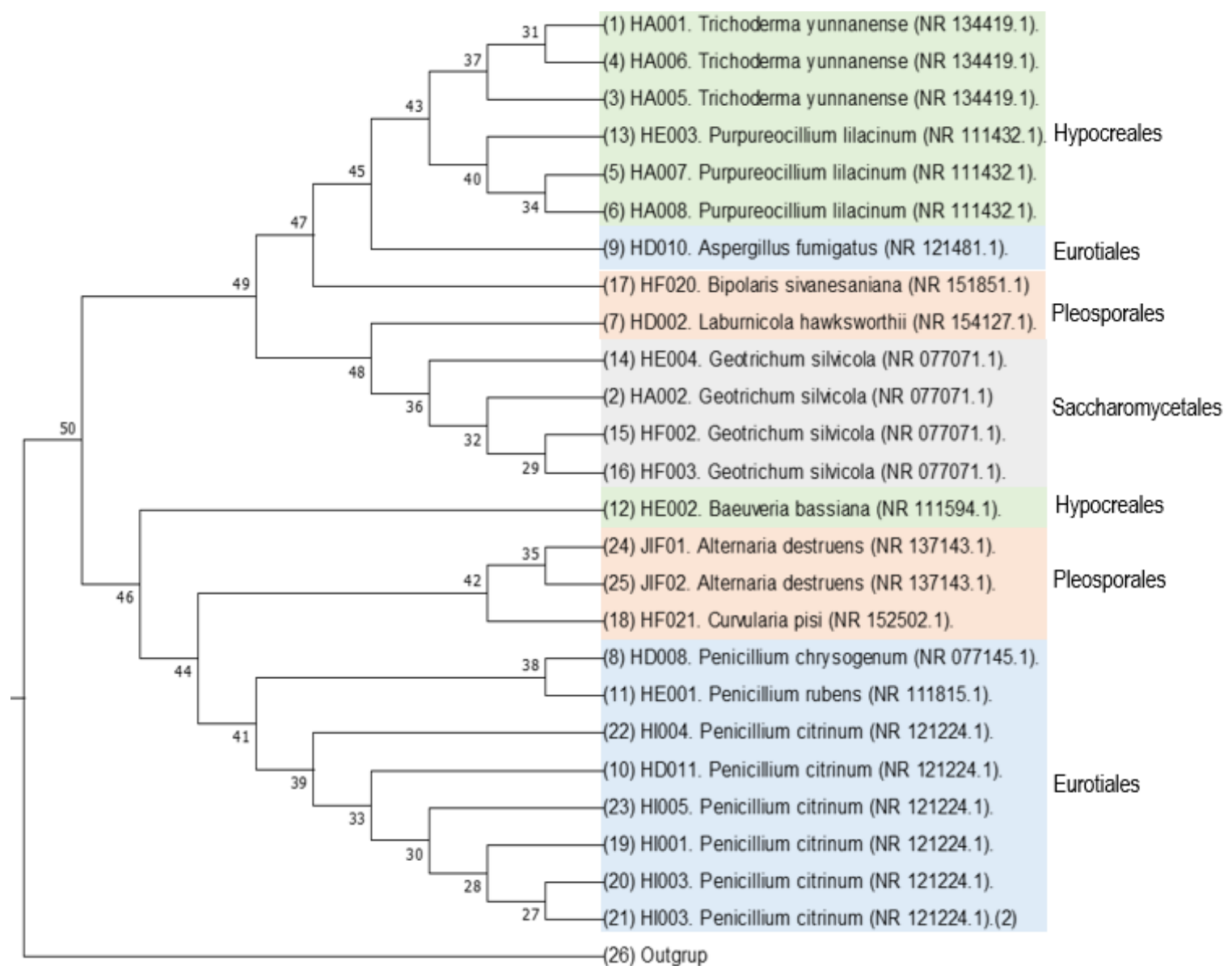

Figura 2. Cladrograma. Representación de la proximidad filogenética de las secuencias amplificadas de la región ITS de las especies identificadas.

A manera de una descripción textual de los resultados representados en el cladograma, se señala lo siguiente: el árbol se dividió en dos grandes ramas que corresponden a los nodos 25 y 50, el nodo 25 se ubicó el grupo externo (outgrup) que se seleccionó de una secuencia de E. coli, mientras que en el nodo 50 se generó la especiación de las 25 cepas identificadas. Del nodo 50 se ramificaron dos nodos superiores, el 49 y el 46 : en el nodo 46 se produjo una bifurcación resultando los nodos 47 y 48 , en el nodo 47 se presentó una especiación directa en el taxón Bipolaris sivanesaniana (nodo 17) y se generó el nodo 45 el cual también se bifurcó en dos clados repre- 
sentados en los nodos 43 y 9 en los que se ubicaron el orden de los Hypocreales y Eurotiales respectivamente; de los Hypocreales surgió la especiación de Trichoderma yunnanense (nodo 37) y Purpureocillum lilacinum (nodo 40) y en el nodo 9 se ubicó Aspergillus fumigatus, el nodo 48 se ramificó en los nodos 7 y 35 ubicando las especies Laburnicola hawksworthii y Geotrichum silvícola, respectivamente. El nodo superior 46 se ramificó en los nodos 12 y 44, en el nodo 12 se encontró el taxón Baeuveria bassiana que pertenece al orden de los Hypocreales, mientras que en el nodo 44 se formaron dos clados que corresponden a los nodos 42 y 41 , en el nodo 42 se ubicaron especies del orden de los Pleosporales pero de los géneros Curvularia y Alternaria, y en el nodo 41 se agrupó el clado que corresponde al orden de los Eurotiales que pertenecen al género Penicillum; a su vez, en el nodo 42 se manifiestó la especiación de Curvularia pisi (nodo 18), y Alternaria destruens (nodos 24 y 25). Curvularia, tradicionalmente ubicada en el género Bipolaris, fue recientemente reclasificada en Curvularia, con base a análisis filogenéticos, incluyendo a B. australiensis, B. hawaiiensis y B. spicifera (Madrid et al., 2019).

Umaña et al. (2019), mediante un estudio de distancias génicas utilizando intermicrosatélites (ISSR), identificó distanciamiento entre cepas de la misma especie, lo que concuerda con lo hallad en esta investigación, pero difiriendo en el método utilizando e identificando diferentes especies. Para el caso de la especie Geotrichum silvícola, las cepas HFOO2 y HFOO3 demuestran mayor proximidad en comparación con las cepas HA002 y HE004; para la especie Trichoderma yunnanense se evidenció que las cepas HA001 y HA006 tienen mayor grado de parentesco en comparación con la cepa HA005; caso similar sucedió con la especie Purpureocillum lilacinum que reflejó un mayor grado de semejanza entre las cepas HA007 y HA008 comparada con la cepa HE003; para el género Penicillumi que presentó variedad de especies identificadas, se evidencia que las especie Penicillum chrysogenum y Penicillum rubens tienen mayor grado de parentesco al agruparse en el mismo nodo, mientras que Penicillum citrum mantuvo un mayor grado de semejanza con los de su misma especie, lo que evidencia que las variaciones genéticas entre aislamientos no dependen de las distancias geográficas (Umaña et al., 2019).

\section{Potencial biotecnológico de los hongos identificados}

Se realizó una recopilación bibliográfica en la que se describieron las capacidades reportadas por otros autores a cerca de cada género y especie de los hongos que se identificaron en esta investigación, buscando el potencial de las cepas para futuras aplicaciones.

Trichoderma es importante por su capacidad de adaptación y producción de metabolitos, como enzimas, compuestos promotores de crecimiento vegetal y compuestos volátiles, entre otros, de interés biotecnológico y ambiental. Este género es utilizado como agente de biocontrol contra hongos fitopatógenos debido a sus múltiples mecanismos de acción, destacando la antibiosis, el micoparasitismo, la competencia por espacio y nutrientes, y la producción de metabolitos secundarios. Varias especies de Trichoderma se han utilizado en sistemas acoplados de fermentación en sustratos sólidos o cultivos sumergidos, para generar energías alternativas como etanol. Los biorreactores como sistema de fermentación optimizan las condiciones del cultivo para favorecer la generación de biomasa y metabolitos (Hernández et al., 2019).

Purpureocillum lilacinum favorece el crecimiento, el contenido de clorofila y controla fitopatógenos como Botrytis que causan enfermedades del moho gris en una gran variedad de frutas y verduras, y la pudrición blanda y tizón foliar en la zanahoria (Rui et al., 2020). P. lilacinum es un nematófago que tiene capacidad ovicida contra Ancylostoma que es un parásito gastrointestinal (Menezes et al., 2017), también tiene la capacidad de ejercer biocontrol contra Meloidogyne incognita que es uno de los patógenos más importantes del pepino (Cucumis sativus L.) y ha demostrado su efectividad en el control de plagas como Meloidogyne javanica, Heterodera avenae Wollenweber, y Radopholus similis que afectan la plantaciones de tomate, cebada y banano respectivamente (Dahlin et al., 2019), además de la capacidad de solubilizar fosforo, producir sideróforos y ácido indol-3-acético (IAA) características de un candidato para uso como biofertilizante (Moreno et al., 2020). 
Geotrichum silvicola es una especie de levadura artroconidial asexual que fue reportada en el año 2005 y, en la actualidad, la información relacionada con el potencial de sus aplicaciones es escasa, pero se ha encontrado que tiene estrecha relación genética con Galactomyces geotrichum, que es una especie con potencial en la industria de alimentos, confiriendo características organolépticas y nutricionales de sabor, aroma y ácidos grasos poliinsaturados (omega 3 y 6) (Grygier et al., 2019).

Se identificaron tres especies del género Penicillum, Penicillum citrium y Penicillum rubens. Son destacados productores de enzimas xilanolíticas, como Penicillium chrysogenum, capaz de metabolizar xilasa que es una enzima que degrada polisacáridos de material celulolíticos y lignocelulósico para producir etanol y bioetanol de segunda generación, también para el tratamiento de jugos, cervezas y vinos, y para producir xilitol (Cabral et al., 2018; Delgado 2021); tiene la capacidad de remediar contaminación con dihidroxibencenos, sulfonatos lineales de alquilbenceno y desperdicios de grasas que son compuestos que actúan como contaminantes de fuentes hídricas (Franco et al., 2020). Penicillum chrysogenum es un generoso productor de penicilina y derivados con potencial terapéutico y de control biológico de hongos, bacterias y virus (Huber et al., 2020), mientras que Penicillium citrinum ha demostrado tener capacidad lipasa, xilanasa y celulasa y de producir quitosano, pencitrin y pencitrinol (Ouephanit et al., 2019; Lina et al., 2019; Tejas et al., 2019; Lodha et al., 2020; Huber et al., 2020).

La primera sustancia antibiótica descubierta fue la Penicilina, por Alexander Fleming, quien observó que el hongo del pan inhibía el crecimiento de las bacterias de S. aureus que había sembrado, llamando al compuesto Penicilina (Penicillium notatum), pero como el efecto inhibitorio era mínimo llegó a pensar que esa sustancia carecía de interés comercial. Se propuso purificar Penicilina de una antibiótica 5-15 cepa que era mejor productora, dando comienzo a la era antibiótica (Asier, 2021).

Aspergillus fumigatus es un reconocido patógeno oportunista, causante de queratitis, reacciones alérgicas en las vías respiratorias y aspergilosis pulmonar (Nayak et al., 2018). También, tiene aplicaciones biotecnológicas por su capacidad de producir lovastatina que es un metabolito secundario fúngico frecuentemente utilizado con fines farmacéuticos para reducir los niveles de colesterol y eventos cardiovasculares, puede producir enzimas con características celulasas, ?-xilosidasa, FPasa, xilanasa, peptidasa y amilasa que tienen aplicación en diferentes sectores productivos como la industria de producción de bioetanol (Xianchun et al., 2020; Mondal et al., 2020). Las celulasas son un conjunto de enzimas hidrolíticas que descomponen la celulosa, mediante la actividad sinérgica de tres diferentes componentes, las endoglucanasa, exoglucanasa y la ß-glucosidasa. A lo largo de los años, estudios de celulasas han demostrado ser potencial biotecnológico en diferentes áreas, aplicada principalmente por ser biodegradables e inofensivas con el medio ambiente. Su producción es bastante estudiada en residuos agrícolas o agroindustriales. La creciente industrialización de estas áreas, ha llevado a la producción de altas cantidades de residuos que no son tratados adecuadamente, siendo importante el aislamiento de microorganismos con potenciales celulolíticos, donde Fusarium sp. CCLM CX presentó la mayor actividad enzimática (Rodríguez, 2019).

Además, se han realizado otros trabajos para evaluar organismos antagonistas para el control de Sclerotinia sp. causante de la podredunbre blanca en lechuga, como, por ejemplo: Trichoderma sp., Aspergillus flavipes, Muscodor yucatanensis, Penicillium commune, Bacillus sp., Clonostachys sp., Paecilomyces lilacinus, Pseudomonas sp. y Coniothyrium sp. (Zubieta et al., 2021).

Baeuveria bassiana es un entomopatógeno que controla plagas de insectos como Nilaparvata lugen, Lasioderma serricorne, Leptinotarsa decemlineata, Plutella xylostella, Phenarium purpurascens, Kuschelorhynchus macadamiae y Carya illinoinensis que atacan los cultivos de arroz, patata, repollo, maíz, macadamia y nuez (Romero et al., 2020; Agboyi et al., 2020; Khun et al., 2020). 


\section{CONCLUSIONES}

Se lograron identificar hongos pertenecientes al género y especies Trichoderma yunnanense, Geotrichum silvícola, Purpureocillium lilacinum, Laburnicola hawksworthii, Penicillium chrysogenum, Aspergillus fumigatus, Penicillium citrinum, Penicillium rubens, Beauveria bassiana, Bipolaris sivanesaniana, Curvularia pisi, y Alternaria destruens. Se mostró una relación taxonómica entre grupos como Trichoderma y Purpureocillum, entre Penicillum, y AlternariaCurvularia, indicando que son géneros filogenéticamente próximos.

La caracterización molecular y la recopilación del potencial biotecnológico para hongos filamentosos permitirá avanzar en proyectos enfocados a la bioprospección, conocer cualidades y mecanismos de estos microorganismos con miras al desarrollo de procesos y productos biotecnológicos, comprender mecanismos de patogénesis y desarrollar alternativas de control de patógenos principalmente para cultivos de interés agrícola y comercial.

\section{AGRADECIMIENTOS}

A Colciencias y al Fondo de Investigaciones de la UFPS (FINU), por la financiación de este proyecto. Al PhD. Andrés F. Barajas.

\section{REFERENCIAS}

AGBOYI-LAKPO, K.; KETOH-GUILLAUME, K.; DOURO-KPINDOU, O.; MARTIN, THIBAUD; GLITHO, I..; TAMÓ, M. Improving the efficiency of Beauveria bassiana applications for sustainable management of Plutella xylostella (Lepidoptera: Plutellidae) in West Africa. Biological Control, v. 144, 2020, p. 104233. https://doi.org/10.1016/j.biocontrol.2020.104233

ALBERTI, FABRIZIO; FOSTER, GARY D.; BAILEY, ANDY M. Natural products from filamentous fungi and production by heterologous expression. Applied Microbiology Biotechnoogy, v. 101, n. 2, 2017, p. 493-500. https://doi.org/10.1007/s00253-016-8034-2

ASIER-DOMINGUEZ, SAN P. Caracterización de microorganismos productores de antibióticos aislados en el proyecto micromundo-UZ [Tesis de Biotecnología]. Zaragoza (España): Universidad De Zaragoza. Facultad de Ciencias, 2021, $52 \mathrm{p}$. https://doi.org/10.1016/j.tifs.2016.10.012

BELLEMAIN, E.; CARLSEN, T.; BROCHMANN, C.; COISSAC, E.; TABERLET, P.; KAUSERUD, H. ITS as an environmental DNA barcode for fungi: an in silico approach reveals potential PCR biases. BMC Microbiology, v. 10, n. 189, 2010, p. 1-9.

https://doi.org/10.1186/1471-2180-10-189

BÉJAR, V.; VILLANUEVA, F.; LEÓN, S.; GUEVARA-GRANADOS, J; URIBE, A.; VERGARAY, G.; CUADRA, A.; SABOGAL, I. Identificación molecular de aspergillus fumigatus aislados de pacientes con aspergilosis invasiva. Revista Peruana de Medicina Experimental y Salud Pública, v. 36, n.1, 2019, p. 81-86. https://doi:10.17843/rpmesp.2019.361.3403

BLANCO-MANZANO, J.; SUAREZ-CONTRERAS, L. Caracterización molecular del fitopatógeno Moniliophthora roreri, utilizando marcadores issr, en Norte de Santander, Colombia. Prospectiva, v.19, n.1, 2021, p. 1-16. https://doi.org/10.15665/rp.v19i1.2234

CENTRO NACIONAL DE INFORMACIÓN BIOTECNOLÓGICA (NCBI) [Internet]. Bethesda (MD): Biblioteca Nacional de Medicina (EE. UU.), Centro Nacional de Información Biotecnológica; 1988. https://www.ncbi. nlm.nih.gov/ [consultado el 16 de septiembre de 2019]. 
DAHLIN, P.; EDER, R.; CONSOLI, E.; KRAUSS, J.; KIEWNICK, S. Integrated control of Meloidogyne incognita in tomatoes using fluopyram and Purpureocillium lilacinum strain 251. Crop Protection, v. 124, 2019, p. 104874. https://doi.org/10.1016/j.cropro.2019.104874

DELGADO-OLIVARES, L. Producción de etanol por digestión de residuos lignocelulósicos debido a hongos del suelo agrícola de la ciudad de lima. PURIQ, v. 3, n.1, 2021, p. 286-302. https://doi.org/10.5598/imafungus.2011.02.01.12

DUFOSSÉ, L.; FOUILLAUD, M.; CARO, Y.; MAPARI, S.A.; SUTTHIWONG, N. Filamentous fungi are large-scale producers of pigments and colorants for the food industry. Current Opinion in Biotechnology, v. 26, 2014, p. 56-61. https://doi.org/10.1016/J.COPBIO.2013.09.007

ESTRADA-SALAZA, GLORIA I.; RAMÍREZ-GALEANO, MARTHA C. Micología general. 1ed. Manizales (Colombia): Centro Editorial Universidad Católica de Manizales, 2019, ISBN 978-958-52337-1-3, 40 p.

FRANCO-COSTA, MAYARA; DE OLIVEIRA, A..; DE OLIVEIRA, E.; DE OLIVEIRA, J. Biodegradation of linear alkylbenzene sulfonate (LAS) by Penicillium chrysogenum. Bioresource Technology Reports, v. 9, 2020, p. 100363. https://doi.org/10.1016/j.biteb.2019.100363

FROESCHKE, G; VON DER-HEYDEN, S. A Review of Molecular Approaches for Investigating Patterns of Coevolution in Marine Host-Parasite. Relationships, v.84, 2014, p. 209-252. https://doi.org/10.1016/ B978-0-12-800099-1.00004-1

GONZÁLEZ-ESTRADA, R..; VEGA-ARREGUÍN, J.; ROBLES-VILLANUEVA, B..; VELÁZQUEZ-ESTRADA, R.; RAMOS-GUERRERO, A.; GUTIÉRREZ-MARTÍNEZ, P. Evaluación in vitro de productos químicos no convencionales para el control de Penicillium citrinum. Polibotanica, v. 49, 2020, p. 161-172. https://doi:10.18387/polibotanica.49.11

GRYGIER, A.; MYSZKA, K.; JUZWA, W.; BIAŁAS, W.; RUDZIŃSKA, M.; ZIŃSK, R. Galactomyces geotrichum mold isolated from a traditional fried cottage cheese produced omega-3 fatty acids. Journal International of Food Microbiology, v. 319, 2019, p. 108503. https://doi.org/10.1016/j.ijfoodmicro.2019.108503

HERNÁNDEZ-MELCHOR, D.; FERRERA-CERRATO, R.; ALARCÓN, A.. Trichoderma: importancia agrícola, biotecnológica, y sistemas de fermentación para producir biomasa y enzimas de interés industrial. Chilean Journal of Agricultural Animal science, v. 35, n. 1, 2019, p. 98-112. https://doi.org/10.1016/j.kijoms.2018.03.002

HUBER, A.; GALGÓCZY, L.; VÁRADI, G.; HOLZKNECHT, J.; KAKAR, A.; MALANOVIC, N.; LEBER, R.; KOCH, J.; KELLER, M.; BATTA, G.; TÓTH, G.; MARX, F. Two small, cysteine-rich and cationic antifungal proteins from Penicillium chrysogenum: A comparative study of PAF and PAFB. Biochimica et Biophysica ActaBiomembranes, v. 1862, n. 8, 2020, p. 183246. https://doi.org/10.1016/j.bbamem.2020.183246

INDEX FUNGORUM. Recuperado el 08 de octubre del 2021 (http://www.indexfungorum.org/names/Names.asp)

JIN, X.; SONG, J.; MA, J.; LIU, GAO-QIANG. Thermostable $\beta$-xylosidase from Aspergillus fumigatus: Purification, characterization and potential application in lignocellulose bioethanol production, Renewable Energy, v. 155,2020 , p. $1425-1431$. https://doi.org/10.1016/j.renene.2020.04.054

KHUN, K.; MASH, G.; STEVENS, M.; HUWER, R.; WILSON, B. . Response of the macadamia seed weevil Kuschelorhynchus macadamiae (Coleoptera: Curculionidae) to Metarhizium anisopliae and Beauveria bassiana in laboratory bioassays. Journal of Invertebrate Pathology, v. 174, 2020, p. 107437. https://doi.org/10.1016/j.jip.2020.107437

LESSICK, B. Johns Hopkins University. Quick guide on using MEGA phylogeny software.(Consultado junio 16 de 2020).

LIMA, R.; ALVES, A.; DE PAULA, A.; DE CASTRO, H.; ANDRADE, G. Mycelium-bound lipase from Penicillium citrinum as biocatalyst for the hydrolysis of vegetable oils, Biocatalysis and Agricultural Biotechnology, v. 22, 2019, p. 101410.

https://doi.org/10.1016/j.bcab.2019.101410 
LIU, R.; KHAN, R.; YUE, Q.; JIAO, Y.; YANG, Y.; LIN, Y.; XIE, B. Discovery of a new antifungal lipopeptaibol from Purpureocillium lilacinum using MALDI-TOF-IMS, Biochemical and Biophysical Researchs Communications, v. 527, n. 3, 2020, p. 689-695. https://doi.org/10.1016/j.bbrc.2020.05.021

LODHA, A.; PAWAR, S.; RATHOD, V. Optimised cellulase production from fungal co-culture of Trichoderma reesei NCIM 1186 and Penicillium citrinum NCIM 768 under solid state fermentation. Journal of Environmental Chemical Engineering, v. 8, n. 5, 2020, p. 103958. https://doi.org/10.1016/j.jece.2020.103958

MADRID, H.; CÁRCAMO, C.; TAPIA, C. Curvularia spicifera. Revista Chilena de Infectología, v. 36, n. 5, 2019, p.646-647. https://scielo.conicyt.cl/pdf/rci/v36n5/0716-1018-rci-36-05-0646.pdf

MANAMGODA, D.; ROSSMAN, A; CASTEBLURY, L.; CROUS, P.; MADRID, H.; CHUKEATIROTE, E.; HYDE, K. The genus Bipolaris. Studies in Mycology, v. 79, 2014, p. 221-288. https://doi.org/10.1016/J.SIMYCO.2014.10.002

MANCILLA, J.; MORA, M.; MONTAÑEZ, D.; MARTÍNEZ, V.; FUENTES, R.; CARRANZA, R. Micobiota aislada de serpientes en cuarentena del centro para investigaciones y respuestas en ofidiología de la Universidad de Panamá (CEREO). Tecnociencia, v. 22, n.2, 2021, p. 180-197. https://doi.org/10.48204/j.tecno.v23n1a10

MENOLLI, N.; SÁNCHEZ-GARCÍA, M. Brazilian fungal diversity represented by DNA markers generated over 20 years. Brazilian Journal of Microbiology, v. 51, 2020, p. 729-749. https://doi.org/10.1007/s42770-019-00206-y

MENEZES-HOFSTÄTTER, B.; DA SILVA-FONSECA, A.; DE SOUZA, F.; DE SOUZA-SILVEIRA, J.; MARONEZEPERSICI. B.; PÖTTER, L.; SILVEIRA, A.; ANTONIOLLI, Z.; BRAYER-PEREIRA, D. Effect of Paecilomyces lilacinus, Trichoderma harzianum and Trichoderma virens fungal extracts on the hatchability of Ancylostoma eggs. Revista Iberoamericana de Micología, v. 34, n. 1, 2017, p. 28-31. https://doi.org/10.1016/J.RIAM.2016.04.004

MONDAL, S.; SOREN, J.; MONDAL, J.; RAKSHIT, S.; KUMAR-HALDER, S.; CHANDRA- MONDAL, K. Contemporaneous synthesis of multiple carbohydrate debranching enzymes from newly isolated Aspergillus fumigatus SKF-2 under solid state fermentation: A unique enzyme mixture for proficient saccharification of plant bioresources. Industria Crops and Products, v. 150, 2020, p. 112409. https://doi.org/10.1016/j.indcrop.2020.112409

MORENO-SALAZAR, R.; SÁNCHEZ-GARCÍA, I.; CHAN-CUPUL, W.; RUIZ-SÁNCHEZ, E.; HERNÁNDEZORTEGA, H.; PINEDA-LUCATERO, J.; FIGUEROA-CHÁVEZ, D. Plant growth, foliar nutritional content and fruit yield of Capsicum chinense biofertilized with Purpureocillium lilacinum under greenhouse conditions. Scientia Horticulturae, v. 261, 2020, p. 108950. https://doi.org/10.1016/j.scienta.2019.108950

NAYAK, A.; CROSTON, T.; LEMONS, A.; GOLDSMITH, W.; MARSHALL, N.; KASHON, M.; GERMOLEC, D.; BEEZHOLD, D.; GREEN, B. Aspergillus fumigatus viability drives allergic responses to inhaled conidia. Ann Allergy, Asthma Immunology, v. 121, n. 2, 2018, p. 200-210. https://doi.org/10.1016/J.ANAI.2018.04.008

OUEPHANIT, C.; BOONVITTHYA, N.; THEERACHAT, M.; BOZONNET, S.; CHULALAKSANANUKUL, W. Efficient expression and secretion of endo-1,4- $\beta$-xylanase from Penicillium citrinum in non-conventional yeast Yarrowia lipolytica directed by the native and the preproLIP2 signal peptides. Protein Expression and Purification, v. 160, 2019, p. 1-6. https://doi.org/10.1016/j.pep.2019.03.012

RAMAKUWELA, T.; HATTING, J.; BOCK, C.; VEGA, F.;WELLS, L"; MBATA, G.; SHAPIRO-ILAN, D. Establishment of Beauveria bassiana as a fungal endophyte in pecan (Carya illinoinensis) seedlings and its virulence against pecan insect pests. Biological Control, v. 140, 2020, p. 104102.

https://doi.org/10.1016/j.biocontrol.2019.104102 
RODRÍGUEZ-ANDACHI, A. Actividad celulolítica de hongos filamentosos aislados del Parque Nacional de Foz de Iguaçu - Brasil en sustratos agrícolas mediante la planificación experimental [Tesis en Biotecnología]. Foz de Iguaçu (Brasil): Universidad Federal de la integración Latinoamericana, 2019, 54 p. https://doi.org/10.1016/J.MICPATH.2017.09.025

ROJAS-PRIETO, N. Evaluación de dos residuos pecuarios en la elaboracion de un biofertilizante empleando fermentación anaerobia [Tesis Magíster en Biotecnología]. Medellín (Colombia): Universidad Pontificia Bolivariana, Escuela de Ingenierías, 2020, 117 p. https://doi.org/10.1016/J.BIOCONTROL.2017.07.001

ROMERO-ARENAS, O.; AMARO-LEAL, L.; RIVERA, A.; PARRAGUIRRE-LEZAMA, C.; SÁNCHEZ-MORALES, P.; VILLA-RUANO, N. Formulations of Beauveria bassiana MABb1 and mesoporous materials for the biological control of Sphenarium purpurascens in maize crops from Puebla, Mexico. Journal of Asia-Pacific Entomology, v. 23, n. 3, 2020, p. 653-659. https://doi.org/10.1016/j.aspen.2020.05.005

SMITH, H.; DOYLE, S.; MURPHY, R. Filamentous fungi as a source of natural antioxidants. Food Chemistry, v. 185, 2015, p. 389-397. https://doi.org/10.1016/J.FOODCHEM.2015.03.134

SUÁREZ-CONTRERAS, L. Identificación molecular de aislamientos de Moniliophthora roreri en huertos de cacao de Norte de Santander, Colombia. Acta Agronómica, v. 65, 2016, p. 51-57. http://dx.doi.org/10.22463/0122820X.1174

SUÁREZ-CONTRERAS, L. Extracción y purificación del AND de Moniliophthora roreri hongo que ataca el cacao, en Norte de Santander. Respuestas, v. 10, n. 2, 2005, p. 4-8. https://doi.org/10.22463/0122820X.629

TÉLLEZ-VARGAS, J.; RODRÍGUEZ-MONROY, M.; LÓPEZ-MEYER, M.; MONTES-BELMONT, R.; SEPÚLVEDAJIMÉNEZ, G.Trichoderma asperellum ameliorates phytotoxic effects of copper in onion (Allium cepa L.). Environmental and Experimental Botany, v. 136, 2017, p. 85-93. https://doi.org/10.1016/J.ENVEXPBOT.2017.01.009

TEJAS-NAMBOODIRI, M.; PAKSHIRAJAN, K. Sustainable and green approach of chitosan production from Penicillium citrinum biomass using industrial wastewater as a cheap substrate, Journal of Environmental Management, v. 240, 2019, p. 431-440. https://doi.org/10.1016/j.jenvman.2019.03.085

UMAÑA-CASTRO, J.; OROZCO-CAYASSO, S.; UMAÑA-CASTRO, R.; MOLINA-BRAVO, R. Identificación molecular y características fisiológica de aislamientos de Trichoderma para el biocontrol de dos patógenos en la piña. Revista de Ciencias Ambientales,Tropical Journal of Environmental Sciences, v. 53, n. 1, 2019, p.125-142. http://doi.org/10.15359/rca.53-1.7

ZUBIETA-CORONADO, D.; ECHEVERRY-PRIETO, L.; ZAFRA-MEJÍA, C. Antagonismo in vitro por consorcios de Trichoderma sp. y Aspergillus sp. contra el fitopatógeno Sclerotinia sp. Biotecnología en el Sector Agropecuario y Agroindustrial, v. 19, n. 1, 2021, p. 16-31. https://doi.org/10.18684/bsaa.v19.n1.2021.1293

WAKAI, S.; ARAZOE, T.; OGINO, C.; KONDO, A. Future insights in fungal metabolic engineering. Bioresource Technology, v. 245. 2017, p. 1314-1326. https://doi.org/10.1016/j.biortech.2017.04.095

WANG, H.; XU, Z.; GAO, L. A fungal phylogeny based on 82 complete genomes using the composition vector method. BMC Evololutionary Biology, v. 9, n. 195, 2009. https://doi.org/10.1186/1471-2148-9-195

WIJAYAWARDENE, N.; HYDE, K.; DAI, D. Outline of Ascomycota, Encyclopedia of Mycology, 2021, p. 246-254. https://doi.org/10.1016/B978-0-12-819990-9.00064-0.

ZHAI, Y.; LI, X.; WANG, T.; WANG, B.; LI, C.; ZENG, G. A review on airborne microorganisms in particulate matters: Composition, characteristics and influence factors. Environment International, v. 113, 2018, p. 74-90.

https://doi.org/10.1016/J.ENVINT.2018.01.007 